\title{
The hypersingular boundary element method revisited
}

\author{
N. A. Dumont \\ Civil Engineering Department, \\ Pontifical Catholic University of Rio de Janeiro, Brazil
}

\begin{abstract}
This is the sequel of a paper presented at the BEM/MRM Conference four years ago, in which the conventional, collocation boundary element method was reformulated by proposing a simple, however consistent derivation on the basis of the weighted-residuals statement. It was shown that the single-layer potential matrix $\mathbf{G}$ should be in general rectangular and satisfy some spectral properties (orthogonality to the space of unbalanced boundary traction forces) in the same way as the double-layer potential matrix $\mathbf{H}$ is orthogonal to rigid-body displacements, when modelling a finite elastic body. Moreover, a "subtle" improvement was proposed for the interpolation of traction forces, in the case of curved boundaries, which was meant to just simplify the numerical implementation. In the present paper, it is concluded that the proposed improvement is in fact a necessary one if strict consistency of the formulation is required and more emphatically if a consistent hypersingular formulation is to be implemented. It is also shown that the correct hypersingular formulation requires that the discontinuous parts (two free terms) of the matrix $\mathbf{H}$ be obtained independently from the matrix G. Motivation of the present developments was the application of the hybrid boundary element method to strain gradient elasticity, which only makes use of the matrix $\mathbf{H}$ together with its hypersingular counterpart. Although this paper is of a rather theoretical nature, a simple numerical example is shown to illustrate the necessity of the proposed improvements.
\end{abstract}

Keywords: boundary elements, weighted-residual methods, hypersingular formulation, curved elements. 


\section{Introduction}

The hypersingular formulation of the collocation boundary element method seems to have been consolidated almost two decades ago with the seminal works done mainly by Guiggiani and some co-workers (as reviewed in Reference [1]) and Mantic and Paris [2], as well as taking into account the conceptual remarks made by Mukherjee [3], to mention just one complementary contribution. Since then, several numerical implementations reported in the literature apparently attest the appropriateness of the proposed developments. However, there still are some conceptual issues to be addressed as a result of the theoretical inquiries carried out starting from variational principles and consistency checks of the classical, collocation boundary element method [4-6]. Motivation of the present developments is a variationally-based application of the boundary element method in the strain gradient elasticity [7,8], from which it turns out that the interpolation function proposed in Reference [5] for the evaluation of the single-layer potential matrix is not just a subtle improvement of the boundary element method, but a decisive requirement when dealing with hypersingularity and curved boundaries. This is particularly necessary if consistency of the formulation regarding rigidbody rotation is to be checked, in a two-dimensional (2D) implementation (since an isoparametric 3D implementation can only be proved fully consistent for a restricted class of boundary elements). The most important conceptual contribution of the present paper is probably the demonstration that the hypersingularity features of the double-layer potential matrix should - and in fact can - be dealt with independently from the corresponding term of the single-layer potential matrix. This sheds light on some hitherto elusive - if not unduly ignored - features of this formulation.

\subsection{Problem formulation}

A simple, consistent derivation of the BEM was presented in Reference [5] and shall not be repeated here. However, it should be restated that one is dealing with the static analysis of an elastic body submitted to traction forces $\bar{t}_{i}$ on part $\Gamma_{\sigma}$ of the boundary and to body forces $b_{i}$ in the domain $\Omega$. Displacements $\bar{u}_{i}$ are known on the complementary part $\Gamma_{u}$ of $\Gamma$. One is looking for an adequate approximation of the stress field that satisfies equilibrium in the domain

$$
\sigma_{j i},{ }_{j}+b_{i}=0 \text { in } \Omega \text {, }
$$

also satisfying the boundary equilibrium and compatibility equations,

$$
\sigma_{j i} n_{j}=\bar{t}_{i} \text { along } \Gamma_{\sigma}, u_{i}=\bar{u}_{i} \text { on } \Gamma_{u},
$$

where $n_{j}$ is the outward unit normal to $\Gamma$. Indices $i, j$, (also $k, l$ ) may assume values 1,2 or 3 , as they refer to the coordinate directions $x, y$ or $z$, respectively, for a general 3D analysis. Sum is indicated by repeated indices. Particularization to $2 \mathrm{D}$ analysis as well as to potential problems is straightforward. 


\subsection{The fundamental solution}

The conventional, collocation boundary element method may be derived for fundamental solutions $\delta \sigma_{i j}^{*}$ and $\delta u_{i}^{*}$ given as

$$
\begin{gathered}
\delta \sigma_{i j}^{*} \equiv \sigma_{i j m}^{*} \delta p_{m}^{*}, \\
\delta u_{i}^{*}=\left(u_{i m}^{*}+u_{i s}^{r} C_{s m}\right) \delta p_{m}^{*},
\end{gathered}
$$

where $u_{i s}^{r}$, for $s=1 \ldots n^{r}$, are $n^{r}$ rigid-body displacements that are multiplied by in principle arbitrary constants $C_{s m}$, and $\delta p_{m}^{*}$ are arbitrary (virtual) force parameters, with $m$ characterizing both location and direction of application of $\delta p_{m}^{*}$. Then, $\delta \sigma_{i j m}^{*}$ and $\delta u_{i m}^{*}$ are functions - with global support - of the coordinates and directions of $\delta p_{m}^{*}$ referred to by $m$ (the source point), as well as of the coordinates and directions referred to by $i$ (the field point), where the effects of $\delta p_{m}^{*}$ are measured.

\subsection{Numerical boundary discretization of displacements and forces}

The displacements $u_{i}$ and the traction forces $t_{i}$ are the problem's unknowns along $\Gamma_{\sigma}$ and $\Gamma_{u}$, respectively. They are approximated along $\Gamma$ as

$$
\begin{gathered}
u_{i}=u_{i n} d_{n}, \\
t_{i}=t_{i \ell} t_{\ell} \equiv\left(|J|_{\text {at } \ell} /|J|\right) u_{i \ell} t_{\ell},
\end{gathered}
$$

where $d_{n}$, for $n=1 \ldots n^{d}$, is a vector of $n^{d}$ nodal displacements and $u_{i n} \equiv u_{i n}(\xi, \eta)$ are interpolation functions with local support, usually piecewise polynomials chosen as functions of the parametric variables $(\xi, \eta)$, for 3D problems, in such a way that, at the nodal points, $u_{i n} \equiv \delta_{i n}$. Since the traction forces $t_{i}$ are surface attributes, the $n^{t}$ parameters $t_{\ell}$ are also surface attributes that depend on the outward normal $n_{i}$ of the boundary point at which $t_{\ell}$ is physically attached. Generally, $n^{t}>n^{d}$, as the boundary $\Gamma$ may not be entirely smooth, with more than one normal at some points. The interpolation functions $t_{i \ell} \equiv t_{i \ell}(\xi, \eta)$ also have local support and should be consistently defined as in eqn (7), where $u_{i \ell}$ are given piecewise as the same polynomials $u_{i n}$ for the displacements of eqn (6), $|J|$ is the Jacobian of the coordinates transformation along the boundary segment and $|J|_{\text {at } \ell}$ is the value of $|J|$ at the nodal point $\ell$, so that $t_{i \ell} \equiv \delta_{i \ell}$ similarly as for the displacements. 


\subsection{Isoparametric formulation and a first consistency check}

The boundary geometry is approximated from the nodal attributes using the same interpolation functions $u_{i n}$ of eqn (10), which consists in an isoparametric representation of the problem. This is expressed as

$$
x_{i}=u_{i n} x_{n},
$$

where $x_{n}$ are nodal Cartesian coordinates and $x_{i} \equiv x_{i}(\xi, \eta)$ are the Cartesian coordinates evaluated for the parametric variables $(\xi, \eta)$. For a generally curved boundary segment expressed according to eqn (7), a general linear displacement field given nodally as $d_{n}^{\text {lin }}$ in eqn (5) is exactly reproduced along $(\xi, \eta)$. This the basis of a $C^{0}$ formulation of the finite element method - a higher order displacement field can be only approximately represented.

For a $2 \mathrm{D}$ problem, the outward unit normal to a boundary segment is given in terms of a single parametric variable $\xi$ by

$$
\left\{\begin{array}{l}
n_{x} \\
n_{y}
\end{array}\right\} \equiv \frac{1}{|J|}\left\{\begin{array}{c}
y^{\prime} \\
-x^{\prime}
\end{array}\right\} \text {, with }|J|=\sqrt{x^{\prime 2}+y^{\prime 2}} .
$$

Since, from eqn (7), $x_{i}^{\prime}=u_{i n}^{\prime} x_{n} \equiv u_{i n} x_{n}^{\prime}$, eqn (8) may also be written in indicial notation in terms of the nodal values $n_{\ell}$ of the outward unit normal as

$$
n_{i}=\left(|J|_{\mathrm{at} \ell} /|J|\right) u_{i \ell} n_{\ell} \quad(2 \mathrm{D} \text { problems }) .
$$

For a 3D problem,

where

$$
\left\{\begin{array}{l}
n_{x} \\
n_{y} \\
n_{z}
\end{array}\right\} \equiv \frac{1}{|J|}\left\{\begin{array}{c}
y_{, \xi} z_{, \eta}-y_{, \eta} z_{, \xi} \\
-x_{, \xi} z_{, \eta}+x_{, \eta} z_{, \xi} \\
x_{, \xi} y_{, \eta}-x_{, \eta} y_{, \xi}
\end{array}\right\},
$$

$$
|J|=\sqrt{\left(y_{, \xi} z_{, \eta}-y_{, \eta} z_{, \xi}\right)^{2}+\left(-x_{, \xi} z_{, \eta}+x_{, \eta} z_{, \xi}\right)^{2}+\left(x_{, \xi} y_{, \eta}-x_{, \eta} y_{, \xi}\right)^{2}} .
$$

Since eqn (10) involves products of derivatives, it is obtained instead of eqn (9)

$$
n_{i} \approx\left(|J|_{\mathrm{at} \ell} /|J|\right) u_{i \ell} n_{\ell} \quad \text { (3D problems, in general). }
$$

It may be checked that this latter equation is only exactly satisfied for linear (threenode) and quadratic (six-node) triangles as well as for the linear (four-node) quadrilateral element.

As a result, a constant stress field can be exactly reproduced according to eqn (6) for any curved 2D boundary element - compare with (9) - whereas this is only conditionally true in the $3 \mathrm{D}$ case. 


\subsection{Basic equation of the collocation boundary element method}

The collocation boundary element method, as given in the literature, is stated as

$$
\left[\int_{\Gamma_{e x t}} \sigma_{j i m}^{*} n_{j} u_{i n} \mathrm{~d} \Gamma+\delta_{m n}\right] d_{n}=\left[\int_{\Gamma} u_{i m}^{*} t_{i \ell} \mathrm{d} \Gamma\right] t_{\ell}+\int_{\Omega} u_{i m}^{*} b_{i} \mathrm{~d} \Omega,
$$

where, for brevity and since it is of no concern for the present developments, an error term that multiplies the constants $C_{s m}$ of eqn (4) is dropped, as compared with the consistent derivation of References [4, 5]. Equation (12) may be written in matrix format as

$$
\mathbf{H d}=\mathbf{G} \mathbf{t}+\mathbf{b},
$$

where $\mathbf{H}=\left[H_{m n}\right] \in R^{n^{d} \times n^{d}}$ is a kinematic transformation matrix, $\mathbf{G}=\left[G_{m \ell}\right] \in R^{n^{d} \times n^{t}}$ is a flexibility-type matrix and $\mathbf{b}=\left[b_{m}\right] \in R^{n^{d}}$ is a vector of nodal displacements equivalent to the applied body forces. The product $\delta \mathbf{p}^{* \mathrm{~T}} \mathbf{H d}$ has the meaning of virtual work, where $\delta \mathbf{p}^{*} \equiv \delta p_{m}^{*}$ are the virtual forces introduced in eqns (3) and (4) [5]. The first motivation for introducing the definition of $t_{i \ell}$ as in eqn (6) was the simplification obtained in the evaluation of $\mathbf{G}$, for curved boundaries, since the Jacobian of $\mathrm{d} \Gamma$ cancels out. It can be now seen, according to the previous Section, that, for a 2D problem with an isotropic material, eqn (13) holds, as stated, exactly for a general linear displacement field, which is not the case of the hitherto proposed boundary element implementations [9]. For a 3D problem, eqn (6) provides only conditionally an exact statement for a linear displacement field, but is nevertheless a simpler and more accurate formulation. The single-layer potential matrix $\mathbf{G}$ is in general rectangular, as stated in eqn (13), since the traction forces depend on the outward normal to the boundary. The fact that $\mathbf{G}$ is rectangular should not hinder the correct proposition of a numerical model and the ultimate evaluation of all relevant quantities of a mechanical problem [9]. The use of different traction forces at a boundary corner leads to locally more accurate results than as in terms of the equivalent nodal forces of the finite element method.

The double-layer and single-layer potential matrices $\mathbf{H}$ and $\mathbf{G}$ comprise in their definition singular and improper integrals, respectively, when source (index $m$ ) and field (index either $n$ or $\ell$ ) refer to the same nodal points. Then, special care must be taken in the numerical integrations. Equation (13) follows the most common developments of the literature, in which the boundary $\Gamma_{\text {ext }}$ encloses the singularity, or source, point characterized by the index $m$. Three mathematically equivalent forms of the matrix $\mathbf{H} \equiv H_{m n}$ are [6]

$$
\begin{aligned}
H_{m n} & =\int_{\Gamma} \sigma_{j i m}^{*} n_{j} u_{i n} \mathrm{~d} \Gamma \equiv \int_{\Gamma_{e x t}} \sigma_{j i m}^{*} n_{j} u_{i n} \mathrm{~d} \Gamma+\delta_{m n} \\
& \equiv \int_{\Gamma_{f p}} \sigma_{j i m}^{*} n_{j} u_{i n} \mathrm{~d} \Gamma+\int_{\Gamma_{d i s}} \sigma_{j i m}^{*} n_{j} u_{i n} \mathrm{~d} \Gamma
\end{aligned},
$$


using $\Gamma$ as the boundary that leaves the source point outside the domain (meaning that the fundamental solution is actually analytical in $\Omega$ ), as illustrated on the left of Figure 1 for the 2D case. The expression used in eqn (13) is the second one both in eqn (15) and in the Figure, with the integration ultimately carried out, as required in a direct numerical evaluation, for the boundary split into a finite part and a discontinuous part, as illustrated on the right of both eqn (15) and Figure 1. The procedures for the numerical evaluation of the finite and discontinuous parts of the integral in eqn (15), for either 2D or 3D problems, is well documented in the literature and shall be not repeated herein.

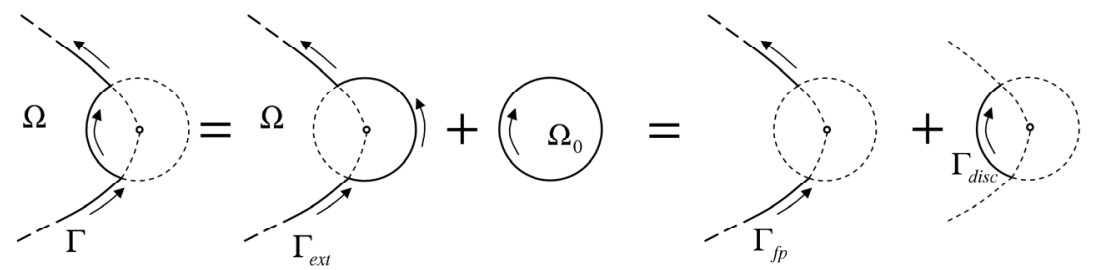

Figure 1: Illustration of the three mathematically equivalent forms of eqn (15) for a bounded domain [5].

\section{Hypersingular implementation of the matrix $\mathbf{H}$}

\subsection{Preliminary developments}

The complete theoretical development of the hypersingular formulation of the collocation boundary element method was primarily formulated by Guiggiani and co-workers, as reviewed in Reference [1], who also credits valuable independent achievements to Mantic and Paris [2]. Their line of reasoning, which intertwines the matrices $\mathbf{H}$ and $\mathbf{G}$ in the evaluation of boundary displacement derivatives in the Cartesian coordinate directions, shall not be reviewed at present. The relevant fact to be brought to light is that, in the variational, hybrid boundary element formulation, use is made only of the matrix $\mathbf{H}$, and a different line of reasoning is necessary when dealing with problems of gradient elasticity, for instance [8]. The following development is proposed for the first time, to the author's best knowledge.

Although not shown before, let $\mathbf{y}$ explicitly characterize the Cartesian coordinates of the source point $m$ (to which the unit point forces $\delta p_{m}^{*}$ of eqns (3) and (4) are applied) and $\mathbf{x}$ be the field point, where the effect of $\delta p_{m}^{*}$ is evaluated and along which integration is ultimately carried out on the boundary eqn (15) this seems to be a common notation in the literature, as given by Guiggiani [1], for instance, although Mukherjee [3] uses exactly the oppose.

The first expression of the double-layer potential matrix $\mathbf{H}$ in eqn (15) is derived - prior to the discretization of the boundary displacements $u_{i}$ as in eqn (5) - with respect to the normal direction $\boldsymbol{\eta} \equiv \eta_{k}$ of the boundary point at which the source is applied: 


$$
\begin{aligned}
\int_{\Gamma} \sigma_{j i m, k}^{*} n_{j} \eta_{k} u_{i} \mathrm{~d} \Gamma & \equiv \int_{\Gamma} \sigma_{j i m, k}^{*}(\mathbf{y}, \mathbf{x}) n_{j}(\mathbf{x}) \eta_{k}(\mathbf{y}) u_{i}(\mathbf{x}) \mathrm{d} \Gamma(\mathbf{x}) \\
& =\int_{\Gamma_{f p}} \sigma_{j i m, k}^{*}(\mathbf{y}, \mathbf{x}) n_{j}(\mathbf{x}) \eta_{k}(\mathbf{y}) u_{i}(\mathbf{x}) \mathrm{d} \Gamma(\mathbf{x}) \\
& +\int_{\Gamma_{d i s c}} \sigma_{j i m, k}^{*}(\mathbf{y}, \mathbf{x}) n_{j}(\mathbf{x}) \eta_{k}(\mathbf{y}) u_{i}(\mathbf{x}) \mathrm{d} \Gamma(\mathbf{x}) .
\end{aligned}
$$

(The Greek letter $\eta$ has already been used to characterize one of the boundary parametric variables for a 3D implementation, as in eqns (10) and (11). However, this latter use, $\eta_{k}$, is indexed, which helps to keep variables apart.) The left-hand side of the above equation uses the compact notation of the paper, whereas the explicit dependence on source and field variables is shown on the right. The integral is also shown as split into a finite and a discontinuous part, following the notation of eqn (15). In allusion to Guiggiani's developments, $\int_{\Gamma_{f p}}$ and $\int_{\Gamma_{\text {disc }}}$ correspond exactly to $\lim _{\varepsilon \rightarrow 0} \int_{\Gamma-e_{\varepsilon}}$ and $\lim _{\varepsilon \rightarrow 0} \int_{s_{\varepsilon}}$, respectively, also using in the following a sphere of radius $\varepsilon$ to characterize the split domains illustrated in Figure 1. Guiggiani [1] does not seem to like resourcing to a finite-part integral, whereas Mukherjee [3] makes some not so clear distinction between a (Hadamard) finite-part integral and a Cauchy principal value. However, as proposed in Reference [10], the finite part of a hypersingular integral can be evaluated entirely on mathematical terms and independently from the concept of a Cauchy principal value.

Next, let $u_{i}(\mathbf{x})$ in eqn (16) be expanded about $u_{i}(\mathbf{y}(\varepsilon=0))$ as a series of $\varepsilon$ :

$$
u_{i}(\mathbf{x})=u_{i}(\mathbf{y}(\varepsilon=0))+\frac{d\left(u_{i}(\mathbf{y}(\varepsilon=0))\right)}{d \varepsilon} \varepsilon+O\left(\varepsilon^{2}\right) \equiv u_{i}(\mathbf{y})+q_{i}(\mathbf{y}) \varepsilon+O\left(\varepsilon^{2}\right) .
$$

In this equation, $\mathbf{y} \equiv \mathbf{y}(\varepsilon=0)$ is explicitly written to emphasize that $u_{i}(\mathbf{y})$ as well as it derivatives are single-valued functions. The normal derivative $q_{i}(\mathbf{y})=d\left(u_{i}(\mathbf{y})\right) / d \varepsilon$ is introduced to simplify notation, but subject to posterior interpretation. Adding and subtracting terms to the integrals of eqn (16), it results

$$
\begin{aligned}
\int_{\Gamma} \sigma_{j i m, k}^{*} n_{j} \eta_{k} u_{i} \mathrm{~d} \Gamma & =\int_{\Gamma_{f p}} \frac{\sigma_{j i m, k}^{*}(\mathbf{y}, \mathbf{x}) n_{j}(\mathbf{x}) \eta_{k}(\mathbf{y}) u_{i}(\mathbf{x}) \mathrm{d} \Gamma(\mathbf{x})}{}+\int_{\Gamma_{\text {disc }}} \sigma_{j i m, k}^{*}(\mathbf{y}, \mathbf{x}) n_{j}(\mathbf{x}) \eta_{k}(\mathbf{y})\left[\underline{u_{i}(\mathbf{x})}-u_{i}(\mathbf{y})-q_{i}(\mathbf{y}) \varepsilon\right] \mathrm{d} \Gamma(\mathbf{x}) \\
& +u_{i}(\mathbf{y}) \eta_{k}(\mathbf{y}) \int_{\Gamma_{\text {disc }}} \sigma_{j i m, k}^{*}(\mathbf{y}, \mathbf{x}) n_{j}(\mathbf{x}) \mathrm{d} \Gamma(\mathbf{x}) \\
& +q_{i}(\mathbf{y}) \int_{\Gamma_{\text {disc }}} \sigma_{j i m, k}^{*}(\mathbf{y}, \mathbf{x}) n_{j}(\mathbf{x}) \eta_{k}(\mathbf{y}) \varepsilon \mathrm{d} \Gamma(\mathbf{x}) .
\end{aligned}
$$

The underlined integral terms are the same ones of eqn (16). The terms in brackets in the second row correspond to the expansion of the displacement $u_{i}(\mathbf{x})$ shown in eqn (17). It is justified to place $u_{i}(\mathbf{y})$ and its derivative, as single-valued functions at $\mathbf{y} \equiv \mathbf{y}(\varepsilon=0)$, outside the integrals of the third and fourth rows. Since the singularity of the product $\sigma_{j i m, k}^{*}(\mathbf{y}, \mathbf{x}) \mathrm{d} \Gamma(\mathbf{x})$ is of order $O\left(\varepsilon^{-1}\right)$ for either a 2D 
or a $3 \mathrm{D}$ problem and the terms in brackets are of order $O\left(\varepsilon^{2}\right)$, according to eqn (17), the whole integral in the second row of the above equation vanishes when $\varepsilon \rightarrow 0$.

The indicated finite-part integral in the first row of this equation means that only the boundary displacement $u_{i}(\mathbf{x})$ is to be interpolated, in the numerical implementation stage, according to eqn (5).

The integrals of the third and fourth rows of this equation are dealt with separately in the following Sections. However, it is worth already noticing the subtle difference in allocating $\eta_{k}(\mathbf{y})$ either outside or inside the integrals. This will be elucidated later on in the frame of a mechanical interpretation. It can be advanced that, in the third row, the nodal displacement $u_{i}(\mathbf{y})$ is independent from the local boundary geometry given by $\Gamma_{d i s c}$, and $\eta_{k}(\mathbf{y})$ just denotes the direction of a fixed, applied virtual double force. On the other hand, $q_{i}(\mathbf{y})$ in the fourth row denotes a displacement gradient that is normal to the boundary, characterized by $\eta_{k}(\mathbf{y})$, which must be considered as varying along $\Gamma_{\text {disc }}$.

\subsection{Evaluation of the integral in the fourth row of eqn (18) for a $2 D$ problem}

In the following, it is shown how to evaluate the term in the fourth row of eqn (18) for the $2 \mathrm{D}$ problem, with results that can be extrapolated to the $3 \mathrm{D}$ case, as carried out in the expanded version of the present paper. In matrix format,

$$
\begin{aligned}
& \sigma_{j i m, k}^{*} n_{j} \eta_{k}= \\
& \frac{-(1-2 v)}{4 \pi(1-v) r^{2}}\left\{\left(2 \frac{\partial r}{\partial \eta} \frac{\partial r}{\partial \xi} \frac{1}{|J|}+\eta_{x} n_{y}-n_{x} \eta_{y}\right)\left[\begin{array}{cc}
0 & -1 \\
1 & 0
\end{array}\right]+\left(2 \frac{\partial r}{\partial n} \frac{\partial r}{\partial \eta}-n_{k} \eta_{k}\right)\left[\begin{array}{ll}
1 & 0 \\
0 & 1
\end{array}\right]\right\} \\
& -\frac{2}{4 \pi(1-v) r^{2}}\left\{\left(4 \frac{\partial r}{\partial n} \frac{\partial r}{\partial \eta}-n_{k} \eta_{k}\right)\left[\begin{array}{cc}
c^{2} & c s \\
c s & s^{2}
\end{array}\right]-\frac{\partial r}{\partial n}\left[\begin{array}{cc}
2 \eta_{x} c & \eta_{x} s+\eta_{y} c \\
\eta_{x} s+\eta_{y} c & 2 \eta_{y} s
\end{array}\right]\right\}
\end{aligned}
$$

with the notation $x / r=c$ and $y / r=s$, for simplicity.

The term $\int_{\Gamma_{d i s c}} \sigma_{j i m, k}^{*} n_{j} \eta_{k} \varepsilon \mathrm{d} \Gamma$ in eqn (18) has the meaning of gradient double tractions that perform virtual gradient work on the gradient displacements $q_{i}(\mathbf{y})$, which are by definition located at the source point $\mathbf{y}$. Then, $\eta_{k}$ must be aligned with $q_{i}$, that is, point outward along the circular exclusion boundary. This justifies writing $\sigma_{j i m, k}^{*} n_{j} \eta_{k} \equiv p_{i m, k}^{*} \eta_{k}$ in polar coordinates for integration over an arch of circle of arbitrarily small radius $\varepsilon$, centered on the singularity point and spanning from $\theta^{-}$to $\theta^{+}$, in terms of the boundary coordinate $\theta$ that substitutes for $\xi$. Since $\quad \mathrm{d} \Gamma=\varepsilon \mathrm{d} \theta, \quad \partial r / \partial n=\partial r / \partial \eta=1, \quad \partial r / \partial \xi=0, \quad n_{x}=\eta_{x}=\cos \theta \equiv \mathrm{c}$, $n_{y}=\eta_{y}=\sin \theta \equiv \mathrm{s}$, the expression of $\sigma_{j i, k}^{*} n_{j} \eta_{k}$ becomes, in polar coordinates, 


$$
\left.\sigma_{j i m, k}^{*} n_{j} \eta_{k}\right|_{d i s c}=\frac{-(1-2 v)}{4 \pi(1-v) \varepsilon^{2}}\left[\begin{array}{cc}
1 & 0 \\
0 & 1
\end{array}\right]-\frac{2}{4 \pi(1-v) \varepsilon^{2}}\left[\begin{array}{ll}
c^{2} & c s \\
c s & s^{2}
\end{array}\right],
$$

and it follows that a corresponding matrix of free terms $C_{i m}$ is expressed as

$$
C_{i m}=\int_{\theta^{-}}^{\theta^{+}} p_{i m, k}^{*} \eta_{k} \varepsilon^{2} \mathrm{~d} \theta=\left.\left(\frac{-\theta}{2 \pi}\left[\begin{array}{ll}
1 & 0 \\
0 & 1
\end{array}\right]-\frac{1}{4 \pi(1-v)}\left[\begin{array}{cc}
c s & -c^{2} \\
-c^{2} & -c s
\end{array}\right]\right)\right|_{\theta^{-}} ^{\theta^{+}},
$$

which is bounded regardless of the value of $\varepsilon$. As it turns out, the integral in the fourth row of eqn (18) leads to the same matrix of free terms as in the case of the classical elasticity - a result that can be extrapolated for the $3 \mathrm{D}$ problem.

\subsection{Evaluation of the integral in the third row of eqn (18) for a 2D problem}

The integrand in the third row of eqn (18) is also evaluated in polar coordinates for integration over an arch of circle of arbitrarily small radius $\varepsilon$, centered on the singularity point and spanning from $\theta^{-}$to $\theta^{+}$, in terms of the boundary coordinate $\theta$ that substitutes for $\xi$, according to the previous Section, although keeping $\eta_{k}(\mathbf{y})$ independent from $\theta$.

As proposed independently by Guiggiani [1] and Mantic and Paris [2] (although the subject is clearer and simpler in Guiggiani's papers), the integral

$$
\lim _{\varepsilon \rightarrow 0} \int_{\theta^{-}}^{\theta^{+}} \frac{f(\theta)}{\varepsilon} \mathrm{d} \theta=\lim _{\varepsilon \rightarrow 0} \frac{b}{\varepsilon}+a,
$$

where $f(\theta)$ is bounded in the integration interval and not supposed to vanish as $\varepsilon \rightarrow 0$, can be formulated as the indicated sum of two terms. The first of them, $b / \varepsilon$, tends to infinity with $\varepsilon \rightarrow 0$ but must cancel with a similarly behaving term in the integral on the right in the first row of eqn (18), such that $\int_{\Gamma} \sigma_{j i m, k}^{*} n_{j} \eta_{k} \delta u_{i} \mathrm{~d} \Gamma$ be ultimately bounded. In fact, the integral on the right in the first row of eqn (18) is evaluated in terms of finite parts, which means that its unbounded part is disregarded only because of its cancelling with $b / \varepsilon$. Then, $a$ is the only term of actual interest in eqn (22). Its evaluation is carried out by expanding a generic integration limit $\theta$ about $\varepsilon=0$ in a Taylor series along the boundary, expressed as

$$
\theta(\varepsilon)=\theta(0)+\left.\frac{d \theta}{d \varepsilon}\right|_{0} \varepsilon+O\left(\varepsilon^{2}\right) .
$$

Since these developments do not present any conceptual difference to Guiggiani's proposition, one may skip further details and summarize that, using

$$
\left.\frac{d \theta}{d \varepsilon}\right|_{0}=\left.\frac{x^{\prime} y^{\prime \prime}-y^{\prime} x^{\prime \prime}}{2|J|^{3}}\right|_{0} \equiv \frac{\left.\kappa\right|_{0}}{2}
$$


where $\kappa$ is the signed curvature of the plane curve $\theta(\varepsilon)$, the evaluation of the finite-part integral (that is, without the infinite term $\lim _{\varepsilon \rightarrow 0} b / \varepsilon$ ) in the third row of eqn (18) can be expressed as the matrix $a_{i m}$ of free terms:

$$
a_{i m}=\eta_{k}(\mathbf{y}) f p \int_{\Gamma_{d i s c}} \sigma_{j i m, k}^{*}(\mathbf{y}, \mathbf{x}) n_{j}(\mathbf{x}) \mathrm{d} \Gamma(\mathbf{x})=-\frac{f_{i m}\left(\theta^{+}\right) \kappa^{+}+f_{i m}\left(\theta^{-}\right) \kappa^{-}}{2},
$$

where

$$
\begin{aligned}
f_{i m}(\theta) & =\frac{(1-2 v)}{4 \pi(1-v)}\left\{\left(\eta_{x} s-c \eta_{y}\right)\left[\begin{array}{cc}
0 & -1 \\
1 & 0
\end{array}\right]+\left(c \eta_{x}+s \eta_{y}\right)\left[\begin{array}{ll}
1 & 0 \\
0 & 1
\end{array}\right]\right\} \\
& +\frac{2}{4 \pi(1-v)}\left\{\left(3\left(c \eta_{x}+s \eta_{y}\right)\right)\left[\begin{array}{cc}
c^{2} & c s \\
c s & s^{2}
\end{array}\right]-\left[\begin{array}{cc}
2 \eta_{x} c & \eta_{x} s+\eta_{y} c \\
\eta_{x} s+\eta_{y} c & 2 \eta_{y} s
\end{array}\right]\right\} .
\end{aligned}
$$

As observed by Guiggiani [1], if the boundary is straight, then $\kappa^{+}=\kappa^{-}=0$; if the boundary is curved, but smooth at the singularity point, then $\kappa^{+}=\kappa^{-}, \vec{\eta}^{+}=\vec{\eta}^{-}$ and $\theta^{+}=\theta^{-}+\pi \Rightarrow f_{\text {im }}\left(\theta^{+}\right)=-f_{\text {im }}\left(\theta^{-}\right)$. In either case, $a_{i m}=0$.

\section{Hypersingular implementation of the matrix $G$}

The single-layer potential matrix $\mathbf{G}$, presented in eqns (13) and (14), has its normal gradient expression - prior to the discretization of the boundary traction forces as in eqn (6) - given in principle by the finite and discontinuous parts

$$
\begin{aligned}
\int_{\Gamma} u_{i m, k}^{*} \eta_{k} t_{i} \mathrm{~d} \Gamma & \equiv \int_{\Gamma} u_{i m, k}^{*}(\mathbf{y}, \mathbf{x}) \eta_{k}(\mathbf{y}) t_{i}(\mathbf{x}) \mathrm{d} \Gamma(\mathbf{x}) \\
& =\int_{\Gamma_{f p}} u_{i m, k}^{*}(\mathbf{y}, \mathbf{x}) \eta_{k}(\mathbf{y}) t_{i}(\mathbf{x}) \mathrm{d} \Gamma(\mathbf{x})+\int_{\Gamma_{d i s c}} u_{i m, k}^{*}(\mathbf{y}, \mathbf{x}) \eta_{k}(\mathbf{y}) t_{i}(\mathbf{x}) \mathrm{d} \Gamma(\mathbf{x}) .
\end{aligned}
$$

This is hitherto a development similar to the one of eqn (16), for the double-layer potential matrix $\mathbf{H}$. However, whereas the displacement $u_{i}(\mathbf{x})$ in the definition of $\mathbf{H}$ is a single-valued function that can be expanded as in eqn (17), the traction forces $t_{i}(\mathbf{x})$ are surface attributes that depend on the outward normal $n_{i}(\mathbf{x})$ to $\Gamma(\mathbf{x})$ and, in the numerical discretization to be adopted, discontinuously defined from boundary segment to boundary segment. This justifies simply ascribing

$$
t_{i}(\mathbf{x})=0 \text { along } \Gamma_{\text {disc }},
$$

with the result that only the finite part of eqn (27) is to be evaluated, although it involves a $r^{-1}$ or $r^{-2}$ singularity for 2D or 3D problems, respectively. 


\section{Conclusions}

As outlined, the double-layer potential matrix $\mathbf{G}$ is rectangular, in a consistent numerical implementation, which also makes use of an improved representation of the traction forces along the boundary, as surface attributes intimately related to the boundary outward normal through the Jacobian $|J|$.

The proposed hypersingular developments treat the single-layer and doublelayer potential matrices $\mathbf{G}$ and $\mathbf{H}$ independently from each other. This is proposed as conceptually superior to the developments in the technical literature. The necessity of such a proposition comes from the variational, hybrid boundary element method, which does not makes use of the matrix $\mathbf{G}$, and thus needs to deal with the matrix $\mathbf{H}$ independently. Moreover, developments in the strain gradient theory of elasticity have led to the present developments. To be emphatic, developments other than the present ones do not lead to verifiably consistent results in gradient elasticity [8].

The singular and hypersingular implementations of the collocation boundary element method can be compactly represented as

$$
\left[\begin{array}{c:c}
\mathbf{H}_{\sigma^{*} d}^{f p}+\mathbf{H}_{\sigma^{*} d}^{\text {disc }} & 0 \\
\hdashline \mathbf{H}_{\sigma^{R} d}^{f p}+\mathbf{H}_{\sigma^{R} d}^{\text {disc }} & \mathbf{H}_{\sigma^{R} q}^{\text {disc }}
\end{array}\right]\left\{\begin{array}{l}
\mathbf{d} \\
\hdashline \mathbf{q}
\end{array}\right\}=\left[\begin{array}{c}
\mathbf{G}_{u^{*} t} \\
\hdashline \mathbf{G}_{u^{R} t}^{f p}
\end{array}\right] \mathbf{t}+\left\{\begin{array}{l}
\mathbf{b}_{u^{*}} \\
\hdashline \mathbf{b}_{u^{R}}
\end{array}\right\},
$$

where the first row of equations is eqn (14) and the matrix $\mathbf{H}$ is represented by its finite and discontinuous parts. The second row of equations shows on the left-hand side $\mathbf{H}_{\sigma^{R} d}^{f p}$ corresponding to the term on the right in the first row of eqn (18), $\mathbf{H}_{\sigma^{R} d}^{\text {disc }}$ corresponding to the term on the third row of eqn (18) and developed in Section 2.3, and $\mathbf{H}_{\sigma^{R} q}^{\text {disc }}$ corresponding to the term on the fourth row of eqn (18) and developed in Section 2.2. The normal displacement gradients $\mathbf{q}$ were introduced in eqn (17) and have the same boundary distribution and orientation of the traction forces $\mathbf{t}$. The matrix $\mathbf{G}_{u^{R_{t}}}^{f f}$ corresponds to the finite part representation of the gradient of $\mathbf{G}$ given in eqn (27), keeping in mind, according to eqn (28), that its discontinuous counterpart is void.

The simplest conceivable numerical verification of eqn (29) is proposed for the example of Figure 2, which simulates an isotropic, elastic body whose strongly curved boundary is given by two quadratic elements [8]. It is checked that the expanded matrix $\mathbf{H}$ of eqn (29) is orthogonal to rigid body translations and rotations. This equation is also exactly checked - within the numerical capacity of representation - for an applied linear displacement field, to which displacement gradients $\mathbf{q}$ and traction forces $\mathbf{t}$ present a strong variation along the curved boundaries. This kind of numerical assessment is more convincing that carrying out a convergence analysis that may just masquerade local errors related to the evaluation of $\mathbf{H}$ and $\mathbf{G}$ for a large number of degrees of freedom. 


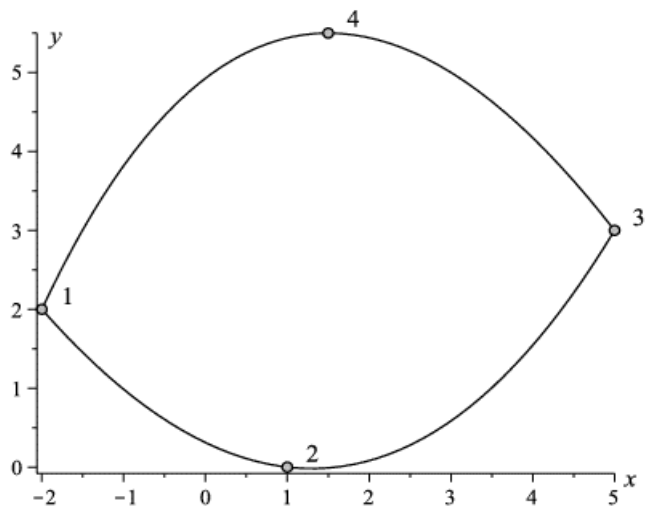

Figure 2: Very simple example with only two quadratic curved elements [8].

\section{Acknowledgement}

This work was supported by the Brazilian agencies CAPES, CNPq and FAPERJ.

\section{References}

[1] Guiggiani, M., Formulation and Numerical Treatment of Boundary Integral Equations with Hypersingular Kernels, eds. V. Sladek and J. Sladek, Singular Integrals in Boundary Element Methods, Chapter 3, 85-117, Computational Mechanics Publications, Southampton, 1998.

[2] Mantic, V.\& Paris, F., Existence and evaluation of the two free terms in the hypersingular boundary integral equations, Engineering Analysis with Boundary Elements, 16, 253-260, 1995.

[3] Mukherjee, S., CPV and HPF integrals and their applications in the boundary element method, International Journal of Solids and Structures, 37, 6623-6634, 2000.

[4] Dumont, N.A., An Assessment of the Spectral Properties of the Matrix G used in the Boundary Element Methods, Computational Mechanics 22(1), 32-41, 1998.

[5] Dumont, N.A., The boundary element method revisited, Boundary Elements and Other Mesh Reduction Methods XXXII, ed. C.A. Brebbia, 227-238, WIT Press, Southampton, 2010.

[6] Dumont, N.A., Assessment of the spectral properties of the double-layer potential matrix H, Boundary Elements and Other Mesh Reduction Methods $X X X V$, eds. C.A. Brebbia and A.H-D. Cheng, 209-219, WIT Transactions on Modelling and Simulation, vol 54, WIT Press, Southampton, 2013. 
[7] Dumont, N.A. \& Huamán, D., A variational boundary element approach for strain gradient elasticity, ICSECM 2013 - The 4th International Conference on Structural Engineering and Construction Management 2013, Proceedings of the Special Session on Advanced Materials, vol 01, 75-89, Kandy, Sri Lanka, 2013.

[8] Dumont, N.A. \& Huamán, D.: A hybrid variational formulation for strain gradient elasticity Part II: boundary element implementation, submitted CMES - Computer Modeling in Engineering \& Sciences, 2014.

[9] Brebbia, C.A., Telles, J.C.F. \& Wrobel, L.C., Boundary Element Techniques, Springer-Verlag: Berlin and New York, 1984.

[10] Dumont, N.A., Simplified assessment and evaluation procedure of finitepart hypersingular integrals, accepted BETeq 2014 - Advances in Boundary Element Techniques XV, Florence, Italy, 2014. 